\title{
Calidad de enseñanza universitaria: relación estudiante/docente
}

\author{
Quality of college teaching: student/docent relationship
}

\author{
Laura Orellana-Cuellar ${ }^{1}$, David Ipenza-Macedo ${ }^{1}$, Joel Coronado-Quispe ${ }^{1}$ \\ ${ }^{1}$ Sociedad Científica de San Fernando. Facultad de Medicina. Universidad Nacional Mayor de San Marcos. Lima, Perú.
}

An Fac med. 2016;77(1):65-6 / http://dx.doi.org/10.15381/anales.v77i1.11557

\section{SR. EDITOR:}

La calidad de educación universitaria en el Perú se rige por el Consejo de Evaluación, Acreditación y Certificación de la Calidad de la Educación Superior Universitaria (CONEAU). La ficha de evaluación de la CONEAU, en la carrera de Medicina, cuenta con 98 estándares de calificación, de los cuales el $N^{\circ} 33$ está relacionado al número de estudiantes por docentes en las teorías y prácticas de laboratorio o prácticas clínicas ${ }^{(1)}$. Durante los últimos años, la cantidad de vacantes ofrecidas de esta carrera se encuentra en aumento. Por este motivo es preciso determinar dicha relación para evaluar la calidad del desarrollo de enseñanza que el estudiante de medicina de la Universidad Nacional Mayor de San Marcos (UNMSM) recibe, de acuerdo a este indicador ${ }^{(2)}$.

Se efectuó una revisión de los syllabus presentados en las asignaturas del primer al quinto ciclo de la EAP de Medicina Humana de la UNMSM. Se trabajó con la cantidad de estudiantes aproximados y la cantidad de docentes de práctica y/o seminario, siendo estos docentes principales, asociados y/o contratados; ambas cifras se encontraban establecidas en el syllabus. Finalmente se procedió a dividir ambas cantidades para establecer la relación estudiante/docente por asignatura. Los resultados son presentados en la tabla 1. En relación a los seminarios, el menor número de alumnos por docente fue de 6 en la asignatura de Ciencias de la Conducta Aplicada a la Medicina y la mayor relación fue de 17 en la asignatura de Sexualidad. Con relación a las prácticas, la menor relación fue de 7 en Patología General y la mayor de 20 alumnos por docente, en las asignaturas de primer ciclo.

El Ministerio de Economía y Finanzas a través del Decreto de Urgencia 033-2005 establece que la relación estudiante/docente no debe ser menor de $12,75^{(3)}$. Así mismo, las cifras de nuestra revisión figuran en el rango de los valores mostrados en las universidades de Reino Unido del tercio superior, que van de 10,3 a 15,3 ${ }^{(4,5)}$. Sin embargo, de acuerdo a Díaz M. en su libro 'Modalidades de enseñanza centradas en el desarrollo de competencias', para optimizar el desarrollo de un grupo de trabajo se recomienda que este debe contar con un número aproximado de 4 a 6 personas, lo que generará que el estudiante tenga un mejor desenvolvimiento en la asignatura ${ }^{(6)}$. Por otra parte, se menciona que a una menor cantidad de estudiantes por docente la enseñanza es personalizada, siendo adecuada para el grupo en el que se busca una parti- cipación horizontal, es decir, un aporte de todos los miembros ejerciendo una retroalimentación del tema tratado ${ }^{(4)}$. En este contexto, se ve cuestionado cuál sería la relación estándar para que el desarrollo del aprendizaje en el estudiante, en base a este indicador de calidad, sea efectivo. Si bien esta relación no influye en la calidad de enseñanza en su totalidad, cuando el límite es mayor se cuenta con un problema de hacinamiento y estrés para el estudiante y el docente.

En nuestra opinión, es preciso evaluar minuciosamente esta situación y establecer una relación adecuada estudiante/docente en seminarios y prácticas, pues esto repercute en el aprendizaje, la metodología del docente, la cohesión del grupo y el seguimiento personalizado de los alumnos.

\section{REFERENCIAS BIBLIOGRÁFICAS}

1. Estándares de Calidad para la Acreditación de Universidades| Sistema Nacional de Evaluación, Acreditación y Certificación de la Calidad Educativa [Internet]. SINEACE | Sistema Nacional de Evaluación, Acreditación y Certificación de la Calidad Educativa. [cited 2015 Jul 30]. Disponible en: http:// www.sineace.gob.pe/acreditacion/universidades/ estandares-de-calidad-para-la-acreditacion-deuniversidades/

2. Jiménez MM, Mantilla E, Huayanay-Espinoza CA, Gil K, Garcia H, Miranda JJ. Demand for training and availability of health science professionals 
Tabla 1. Número de alumnos por docente de práctica y/o seminario.

\begin{tabular}{|c|c|c|c|c|c|c|}
\hline \multirow{2}{*}{$\begin{array}{c}\text { Ciclo } \\
\text { Académico }\end{array}$} & \multirow[b]{2}{*}{ Asignatura } & \multirow{2}{*}{$\begin{array}{l}\text { Estudiantes } \\
\text { matriculados aprox. }\end{array}$} & \multicolumn{2}{|c|}{ Seminario } & \multicolumn{2}{|c|}{ Prácticas } \\
\hline & & & Docentes & $\begin{array}{c}\text { Relación } \\
\text { alumnos docente }\end{array}$ & Docentes & $\begin{array}{c}\text { Relación } \\
\text { alumnos docente }\end{array}$ \\
\hline \multirow{5}{*}{$1^{\mathrm{a}}$} & Biofísica & 160 & 13 & 12,31 & 8 & 20 \\
\hline & Química & 150 & 13 & 11,54 & 6 & 20 \\
\hline & Ética & 150 & 30 & 5 & - & - \\
\hline & Biología & 160 & - & - & 6 & 20 \\
\hline & Ciencias sociales & 150 & 15 & 10 & - & - \\
\hline \multirow{4}{*}{$2^{\mathrm{a}}$} & Conocimiento científico e investigación & 160 & - & - & 19 & 8,42 \\
\hline & Histología & 160 & - & - & 17 & 9,41 \\
\hline & Embriología y genética & 170 & - & - & 12 & 14,17 \\
\hline & Anatomía humana & 160 & - & - & 16 & 10 \\
\hline \multirow{4}{*}{$3^{\mathrm{a}}$} & Fisiología & 145 & 15 & 12,31 & - & - \\
\hline & Bioquímica & 214 & 18 & 11,89 & - & - \\
\hline & Estadística médica y demografía en salud & 160 & 10 & 16 & - & - \\
\hline & Ciencias de la conducta aplicada a la medicina & 170 & 28 & 6,07 & - & - \\
\hline \multirow{5}{*}{$4^{a}$} & Patología general & 160 & 12 & 13,33 & 24 & 6,67 \\
\hline & Inglés I - II & 170 & - & - & & \\
\hline & Microbiología & 180 & - & - & 26 & 6,92 \\
\hline & Parasitología básica & 180 & - & - & 13 & 13,85 \\
\hline & Proyectos de investigación & 150 & - & - & 15 & 10 \\
\hline \multirow{4}{*}{$5^{a}$} & Farmacología & 150 & 15 & 10 & - & - \\
\hline & Fisiopatología & 150 & - & - & - & - \\
\hline & Sexualidad & 136 & 8 & 17 & - & - \\
\hline & Comunitaria & 160 & 16 & 10 & - & - \\
\hline
\end{tabular}

in Peru. Rev peru med exp salud publica. 2015 Mar;32(1):41-50.

3. Beltrán Barco Arlette. Guía de identificación, formulación y evaluación social de proyectos de universidades a nivel de perfil [Internet]. Ministerio de Economia y Finanzas; 2007. Disponible en: http://www.mef.gob.pe/contenidos/inv_publica/ docs/instrumentos_metod/educacion/GuiaUniversidades.pdf

4. Court Stephen. An analysis of student:staff ratios and academics' use of time, and potential links with student satisfaction [Internet]. 2012. Dispo- nible en: http://www.ucu.org.uk/media/pdf/p/p/ ucu_ssranalysis_dec12.pdf

5. Top UK University League Tables and Rankings 2016 [Internet]. [cited 2015 Sep 1]. Disponible en: http://www.thecompleteuniversityguide.co.uk/ league-tables/rankings?o=Student-Staff $\% 20$ Ratio

6. De Miguel Díaz M. Modalidades de enseñanza centradas en el desarrollo de competencias. Orientaciones Para Promover El Cambio Metodol En El Espac Eur Educ Super Oviedo Univ Oviedo. 2005;109-14.
Carta al Editor recibida el 3 de septiembre de 2015.

Fuente de financiamiento: autofinanciado.

Conflictos de Interés: Ios autores declaran no tener conflicto de interés en la publicación de este artículo.

\section{Correspondencia:}

Laura Rocio Orellana Cuellar

Jr. Adolfo Jacobo Mz. Q lote 5 - SMP. Lima, Perú

Correo-electrónico: laura.orellana.1993@gmail.com 